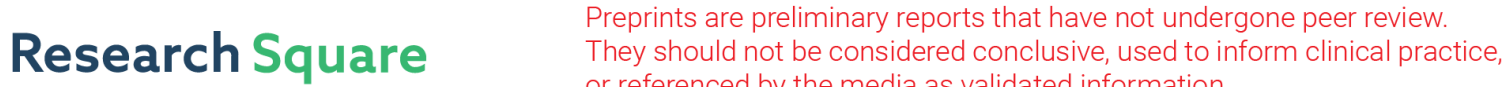 or referenced by the media as validated information. \\ Development the Flexible Magnetic Abrasive Finishing Process by Transmitting the Magnetic Fields
}

abbas moghanizadeh ( $\sim$ abbas.moghanizadeh@gmail.com ) azad university

\section{Research Article}

Keywords: Magnetic Abrasive Finishing, Surface Roughness, Magnetic Field, abrasive particles, magnetic coil

Posted Date: February 10th, 2021

DOl: https://doi.org/10.21203/rs.3.rs-167235/v1

License: (c) (1) This work is licensed under a Creative Commons Attribution 4.0 International License. Read Full License

Version of Record: A version of this preprint was published at The International Journal of Advanced Manufacturing Technology on December 2nd, 2021. See the published version at https://doi.org/10.1007/s00170-021-08269-8. 


\title{
Development the Flexible Magnetic Abrasive Finishing Process by Transmitting the Magnetic Fields
}

\author{
Abbas Moghanizadeh \\ Department of Materials Engineering, Isfahan University of Technology, Iran \\ Corresponding author: abbas.moghanizadeh@gmail.com
}

\begin{abstract}
The objective of this research is to present a novel approach in magnetic abrasive finishing to improve its potential for creating different finishing patterns in free-form surface using no special fixtures or tool machines to minimize the complexity of the process. The key point of this idea is that magnetic abrasive particles can move in especial patterns by transfer magnetic fields (similar a magnetic train moving on a magnetic rail) and create the desired polishing patterns on the surface simultaneously. The coils are placed under a thin plate, then a flexible magnetic path is created by a special arrangement of magnetic coils, after that, the coils are turned on and off in turn, and the magnetic abrasive particles move in the created path and abrasive the surface. The continuous movement of magnetic abrasive particles under the magnetic field will lead to abrasive the surface of thin sheets. The tests were performed on copper sheets with a thickness of $1 \mathrm{~mm}$. Experimental parameters include electric current $(0.25,0.5$, and $0.75 \mathrm{~A})$, speed of turning on and off of the coils (speed of magnetic abrasive particle movement) $(20,30$, and $40 \mathrm{~mm} / \mathrm{s}$ ), and process time (1, 2, and 3 hours). The experiments were performed on an L-shaped and free-form sheets. The results show that using a transmission magnetic field in the MAF (TMAF) makes it easy to create different surface roughness patterns in different directions simultaneously. While in one part of the L shape the electric current is $0.25 \mathrm{~A}$, the surface roughness of is around $0.9 \mu \mathrm{m}$, in the other part, where the electric current is $0.75 \mathrm{~A}$, the surface roughness of is around $0.55 \mu \mathrm{m}$.
\end{abstract}


Meanwhile, TMAF makes it possible to finish a free-form surface with no special fixtures. Moreover, there is a direct relationship between the change in the surface roughness and the electric current and process time.

Keywords: Magnetic Abrasive Finishing; Surface Roughness, Magnetic Field, abrasive particles, magnetic coil.

\section{Introduction}

\subsection{MAF principle}

The magnetic abrasive finishing (MAF) process was introduced by American and Russian researchers[1]. The MAF process is one of the significant non-traditional metal finishing processes by employing magnetic fields and magnetic abrasive particles[2]. In the MAF process, a magnetic field is used to force abrasive particles against the target surface; furthermore, these magnetic abrasive particles can move on the surface of workpiece while the magnetic field bonds them to the surface. In the MAF technique, cutting tools consists of iron and abrasive particles[3]. In addition, the abrasive particles can be mixed with small amounts of metalworking fluid such as distilled water or kerosene to retain the abrasive which adds lubricity and cools the parts[4].

\subsection{MAF conventional equipment}

To apply the MAF technique, a machine tool, abrasive particles, medium composition, and process settings are essential. In many types of research, conventional production machines such as lathe and milling have been utilized for the MAF process according to the workpiece geometries. In the case of MAF for cylindrical surfaces, a lathe can typically be used. Cylindrical parts are typically chucked in a small lathe. In the case of MAF for flat surface, by using milling machines, the magnetic tool is chucked in the spindle and rotated which the magnets are placed a few millimeters above the parts. Meanwhile, a number of researchers introduce special MAF machines to finish complex workpieces by developing freecurved tools which are inserted into the spindle or using robot arms[5]. While few 
researches utilize permanent magnets[6], others prefer to use electromagnetic inductor which consists of an inductor of steel road wrapped with a coil of wire[7].

\subsection{MAF parameters}

Several parameters play a key role in the MAF. Numerous studies show that magnetic field has a strong influence on the finishing process; in addition, the voltage (DC) applied to the electromagnet (in non-permanent magnet), working gap, the rotational speed of the magnet, abrasive size (mesh number) has a significant effect on the MAF process. The experimental findings determine the correlation between surface finishing and the magnetic forces[8]. It is reported that the current value is imposed on the electromagnet (or magnetic flux density) and the reduction in the working gap has a significant effect on the surface roughness $(\Delta \mathrm{Ra})[8]$.

\subsection{MAF applications}

The MAF has unique potential in some applications such as finishing the inner of tubes, free-form surfaces as well as eliminating the side effects of other finishing processes. One of the main applications of the MAF technique is to finish the inner of the tubes. The inner roughness of tubes has a significant effect on the system performance; however, improving their surface roughness is difficult using the conventional finishing method. Meanwhile, using the MAF system can be used to produce good surface quality efficiently[9]. Wang and Hu described the principle of the process and finishing characteristics of unbounded magnetic abrasive to finishing the internal of a tube. Wang studies the effect of internal magnetic abrasive finishing of thin and long austenitic stainless steel tubes[10]; also in addition, improving the internal surface finishing of the bent tube made of stainless steel (SUS304) by using the MAF process has been studied by[11]. Besides finishing inner tubes, the MAF system has a unique potential to improve the finishing of free-form surface operations[12] which are required for some industrial applications such as biomedical, and special surface patterns. For instance, the femoral component of a knee prosthetic requires a specific surface pattern and a level of roughness to function properly. In a case study, Yamaguchi tries to develop the MAF process to improve the surface roughness of a knee prosthetic (free-form) by the complex system using a robot[5]. In addition, some researchers try to develop a polishing of free-form surfaces using different heads. They concluded that the soft polishing head is more efficient than the hard one to finish the free form surface[13]. In addition, 
the MAF achieves to improve surface functionality and the desired values of surface parameters such as wettability, corrosion resistance, and friction which plays an important role in many industrial applications such as optics[14], medical components[15], micro electromechanical systems, electronic components[16]. Moreover, it removes micro-cracks, recast metal, or even visible scratches or damaging residual stresses[16]. While grinding, laser machining, conventional cutting, and even polishing, these unwanted side effects can be produced; as a result, improving the MAF technique potentials will be beneficial for a wide variety of industrial finishing projects.

\subsection{Limitation of conventional MAF}

There are several limitations in applying the MAF technique. Depending on using special tools for movement the magnetics, set some restrictions to MAF and is considered as one of its disadvantages. Using conventional machine tools (lathe and milling) reduce the flexibility of this method. In addition, to finish the surface of complex workpieces such as spheroid, cycloid, cylindroid, and free-curved, special tools or fixtures should be manufactured and inserted into the spindle which increases the cost of the MAF process. Meanwhile, using robots or CNC machines are inevitable for finishing the free-form surfaces automatically which makes this process more complex and expensive. The vibration of machine tools, transmit to the workpiece and lead to a lot of challenges, that is not suitable for the brittle workpiece.

\section{The principle of proposed MAF technique: transmitting magnetic field in magnetic abrasive finishing (TMAF)}

In the current research, a new MAF technique is developed to increase the flexibility of this technique. The key point of this idea is that magnetic abrasive particles follow the transmitting of magnetic fields (similar a magnetic train moving on a magnetic rail). The principle of this technique (as shown in Figure 1) is that the magnetic field, which is produced by electromagnetic coils, transmits from one to another coil, which are placed in special arrangement under a thin sheet; Firstly, the coils are prepared and then mounted on the insulating surface in several rows and below the workpiece. An electrical circuit turns on or off coils according to with the finishing pattern (the current of each coil be controlled individually). It is not necessary to change the physical pattern of coils so that using them is not time- 
consuming. Subsequently, the magnetic field flows in a special path (based on coils arrangement) by turning on and off the coils controlled by an electronic circuit. Afterward, the magnetic force (produced by special coils arrangement) determines the path of the abrasive particle movement and finishes the surface of workpieces.

The magnetic field is created by a coil in which an electrical current flow in the wire around the core as shown in Figure 2; several electro magnet coils are placed next to each other. They can be arranged in many different forms based on the geometry of workpieces or desired special pattens. Electrical current flows to the electromagnetic coils in turn; thus it creates a magnetic field in each coil. An electrical circuit controls the electrical flow and turns on or off the coils in turn; therefore, the generated magnetic field is transmitted from one coil to another (each coil becomes a magnet in turn) and creates a flow of magnetic fields. The magnetic fields which are generated and transmitted by coils bound the abrasive particles on the surface and move them in special directions. Due to the special arrangement of electromagnetic coils, transmitting a magnetic field from one coil to another creates a special path that makes magnetic abrasive particles move based on special patterns created by electromagnetic coils arrangements. The schematic of the process is shown in Figure 1. The abrasive particles can be moved in the $\mathrm{x}, \mathrm{y}$, and/or $\mathrm{z}$ directions with respect to the magnetic field. The movement of the abrasive particles with respect to the arrangement of magnet coils can reduce the surface roughness (Ra) of the workpiece and creates special patterns on the surface of the workpiece.

\section{Experimental Setup}

The main elements of TMAF equipment include the electromagnet, magnetic abrasive particles, and electronic circuits.

\subsection{Electromagnet setup}

Several electromagnetic coils are designed and manufactured to implement magnetic force on the workpiece. A magnetic core is a piece of magnetic material used to confine and guide magnetic fields. It is made of iron alloy along with silicon. To boost the efficiency of the magnetic field, the cores consist of lamination plates. In this study, the thickness of each plate is $1 \mathrm{~mm}$ and there are 10 plates in each core; moreover, the cores are designed in the form of I and the wire diameter is $0.25 \mathrm{~mm}$ as shown in Figure 2. Each coil with around 8000 turns (800 turns per centimeter). 


\section{- Electronic circuits}

An electronic circuit is designed to turn off and on the electrical current on the coils in turn. The designed electronic circuit has the potential to change the speed of turning coils off and on.

\section{- Arrangement the coils}

The coils are glued on an opposite side of plate to arrange the coils for creating finishing L-shape and free form patterns (there is no distance between coils and plate surface).

\subsection{Materials and equipment}

- Magnetic abrasive particles

Abrasive materials consist of Iron powder (the particle sizes are lower than $60 \mu \mathrm{m}$ ) and Aluminum oxide powder (the particle sizes are lower than $10 \mu \mathrm{m}$ ) with a weight ratio of $60 \%$ iron powder and $40 \%$ aluminum oxide powder.

\section{-Workpiece materials}

The plates made of copper with the thickness of $1 \mathrm{~mm}$ are used for the experiments as the workpieces. Meanwhile, a free-form plate is used having the same thickness.

\section{- Surface roughness measuring}

After each experiment, the change in surface roughness value $(\mathrm{Ra})$ is determined by measuring $(\mathrm{Ra})$ via tester TR220. The initial surface roughness (Ra) of the samples is varied and is nearly $2 \mu \mathrm{m}$.

\subsection{Experimental settings}

The following three effective parameters have been taken to conduct the experiment on the TMAF.

- Finishing Time

The process times are 1, 2 and 3 hours.

\section{- Abrasive particles speed}


The average speed of abrasive particles is measured by measuring the time between off and on coils and the distance between the centers of two coils. However, the speed is, in principle, accelerated (after the coil is turned off, the abrasive particles move to the next coil and then stop at that center of the second coil (coil is on)). Nevertheless, we measured the average speed. (Magnetic coils are turned off and on in turn to produce the speed of 20,30 , and $40 \mathrm{~mm} / \mathrm{s}$ for abrasive particles).

\section{- The current of electromagnetic induction}

Electrical current levels including $0.25,0.5$ and 0.75 ampere are used in coils to create different magnetic field strength.

The experiments are conducted to analyze the performance of the transmitting magnetic field in the MAF (TMAF) process. The finishing process is control by adjusting 3 input parameters (time of finishing, particle speed, and electrical current). It should be mention that the TMAF is applied in two manners; first, for flat surfaces enabling two paths (directions) to be finished simultaneously (L-shape) and second, for free-form surfaces (all tests repeated five times).

\section{Results and Discussions}

The experimental results show that the surface morphology of the plates has changed significantly after the TMAF process. Atomic Force Microscope (AFM) images confirm that the surface morphology has improved after the TMF process as shown in Figure 3 and 4. Figure 3 shows that the surface morphology is improved with increasing process time. Furthermore, Figure 4 also shows that with increasing electrical current in the coils, the surface morphology is improved and the surface roughness is reduced (the height of peaks and valleys is reduced).

The reason for the improvement of the surface morphology can be related to the magnetic force applied to the magnetic abrasive particles (the electric current in the coils creates a magnetic field, which leads to applying the magnetic force on magnetic abrasive particles) as shown in Figure 5.

The magnetic field strength at the center of a coil is[17]:

$$
B=\frac{\mu_{0} N I}{L}
$$

where $\mathrm{N}$ is the number of turns of the coil, $\mathrm{L}$ and $\mathrm{I}$ are the length and the current in the coil respectively, and $\mu_{0}=4 \pi \times 10-7 \mathrm{H} / \mathrm{m}$ is the magnetic permeability. 
The magnetic force is proportional to the gradient of the magnetic field as express below[18]:

$$
F_{V}=\frac{\mu_{0}}{2} \nabla \int_{V} M . H d V
$$

where $\mathrm{V}$ is volume of the magnetic abrasive particle, $\mathrm{M}$ and $\mathrm{H}$ are the magnetization and the flux intensity respectively.

The magnetic force $F_{V}$ can be calculated from the equation[19]:

$$
F_{V}=\mu_{0} V(M . \nabla) H
$$

The vertical magnetic force can be considered as:

$$
F_{V}=\frac{\mu_{0} H^{2} A}{2}=\frac{B_{0}^{2} A}{2 \mu_{0}}
$$

where $\mathrm{A}$ is the contact area of the magnetic abrasive particles with the target surface. The magnetic field applies pressure on the surface of magnetic particles. The magnetic field, apply a vertical force to the magnetic abrasive particles on the sheet surface. Then applies forced leads to penetrating magnetic abrasive particles onto a surface, eventually, the movement of thousands of particles will lead to the cutting and shaving of the surface peaks.

Therefore, as the electric current in the coil increases, the intensity of the magnetic field increases (based on Eq. (1)), and as a result, the magnetic force exerted on the abrasive particles will be enhanced (based on Eq. (4)), and the surface morphology will be improved (Figure 4). Also, increasing the TMAF processing time leads to more abrasive particles in contact with the surface and more scratches on the surface peaks and improves the surface morphology (Figure 3).

\subsection{Creating similar surface roughness in the two directions simultaneously by the TMAF}

Having the goal of polishing two perpendicular directions ( $\mathrm{L}$ pattern) simultaneously, (two paths that are perpendicular) magnetic coils are arranged in two perpendicular directions which create an L pattern (by the arrangement of coils) as shown in Figure 7, 8 and 9. Therefore, magnetic fields propagate to electromagnetics in turn and the mixture of magnetic abrasive particles moves along the surface of the workpiece in response to the magnetic field ( $\mathrm{L}$ pattern) and improves the roughness of the surface in harmony with having $\mathrm{L}$ pattern. The measurements show that the surface roughness $(\mathrm{Ra})$ in two directions (path A-B and path B-C) are the same for different process parameters as shown in Figures 7, 8, and 9. It is evident from the figures that transmitting magnetic fields have the same effect on the two directions. This could be due to the magnetic force exerted on the magnetic abrasive particles. Although magnetic abrasive particles move in two 
perpendicular paths (paths of A-B and B-C), due to the arrangement of the coils, the magnetic field strength of the two paths is similar (because the electric current in the coils is the same in both paths, so the magnetic field strength of the two paths is the same). Therefore, the magnetic force exerted on the magnetic abrasive particles is also constant along both paths and as a result, the surface roughness is the same in both paths. This strategy (same current in coils of paths) makes it possible to create surface roughness in various paths without the use of special tools or fixtures and even robot arms. And it is technically feasible to abrasive different paths with the same quality just by arranging different coils, which can reduce the cost of the machining process and make it more flexible. Also, as shown in Figure 7,8 and 9, by selecting the test parameters, the desired surface roughness can be created.

\subsection{Texturing with the TMAF}

Creating different surface roughness is vital for some applications; thus the magnetic force for the bound abrasive particles to the surface should be different. As the current of each coil can be controlled individually, it is possible to create a different magnetic field in each coil. It assists to press magnetic particles to the surface having different magnetic forces. In this case, we generate different magnetic forces in the two preopercular paths (path A-B and path B-C) by increasing the electrical current in the coils located in the path B-C compared with the path A-B ones (electrical current in coils located in path $\mathrm{B}-\mathrm{C}$ is more than path A-B ones). As a result, the magnetic field strength in the path $\mathrm{B}-\mathrm{C}$ is higher than the path $\mathrm{A}-\mathrm{B}$. As expected, the surface roughness in the path B-C with the higher magnetic field is lower than the path A-B as shown in Figures 10 and 11. As mentioned earlier, this is due to the magnetic force exerted on the magnetic abrasive particles. The magnetic field strength and the force exerted on the abrasive particles are proportional to the electric current of the coil (based on Eq. $(1,4)$ ). In a particular path (path B-C), an increase in the electric current of the coils leads to an increase in the magnetic field, and increasing the magnetic field leads to increasing the magnetic force applying on the magnetic abrasive particles and ultimately improving the surface roughness. Therefore, by changing the current intensity in the coils in different paths, different surface roughness can be created. Obviously, Ra reduces with increased inductor current, increased particle speed, and increased finishing time. Increasing the speed of abrasive particles and process time causes more abrasive particles in contact with the surface, which ultimately leads to improved surface smoothness. 
This technique improves the performance of the MAF when the different surface patterns for complex-shaped metal parts are desired. In other words, drafting different surface patterning and smoothing in different directions simultaneously is the ability of this method. Besides, it is clear that conventional machine tools are not required and vibrations in the machining center and machining tool are not transmitted onto the workpiece surface; thus, it can efficiently finish ceramics, carbides, coated carbides, and silicon. Moreover, all functions can be controlled by electric and electronic units which makes the MAF process more appropriate for automation production lines. Evidently, automating the finishing process leads to reducing production costs while ensuring quality control. Meanwhile, it can be deduced from the results that Ra reduces with increased three factors of the inductor current, particle speed, and finishing time. Electrical current is found to be a significant parameter. It is clear from the results that an increase in magnetic flux density leads to reducing in surface roughness. The current input to the coils of the electromagnet generates the magnetic field, which controls the magnetic force that bonds the magnetic particles on the workpiece. In other words, the magnetic flux density dictates the finishing force that is governed by the electrical current in coils. Increasing the density of the magnetic flux causes magnetic particles to stick to the surface of the workpiece more intensely, then the normal magnetic force cutes the peaks of the workpiece surface (when electrical current values grow up, the vertical normal force is dominant and increases indentation of abrasive in workpiece surface and causes an increase in percentage change in surface roughness). Therefore, Shifting the electrical current from low level to high level leads to enhancing the magnetic flux density in coils.

It can be seen from the results that the improvement in surface roughness increases by enhancing the velocity of higher magnetic particle movement. Material removal is essential for improving the surface roughness. The rate of material removal depending on the relative motion between the magnetic abrasive particles and the workpiece. The faster motion of magnetic particles leads to higher surface roughness. The improvement in surface roughness can be due to more abrasive particles that come in contact with the workpiece during high speed because more cutting edges of magnetic particles available per unit of time, which removing the micro and Nano hills and valleys effectively to give greater change in surface roughness. At the same time, the number of cutting edges increases by increasing 
the speed of particles. At the higher speed of particles, the horizontal force is also large and the chances of abrasive particles to indent into the workpiece surface and breakdown the micron hills of the surface increased. This may result in a reduction in percentage change in surface roughness.

\subsection{Finishing of free-form surface by the TMAF}

Having the aim of improving the surface roughness of the free-form surface, the magnetic coils configurations are adopted using the surface curves as shown in Figure 12 (all coils are in parallel). The surface roughness ( $\mathrm{Ra}$ ) of a free-form surface and a flat plate made of copper are compared as shown in Table 1. As expected, the proposed TMAF technique enables us to reduce the roughness of the free-form surface as the same as the flat plate. As shown in Table 1, with the change of process parameters, the surface roughness also changes, which is due to the change of the force exerted on the abrasive magnetic particles, but in all conditions, the surface roughness of the free and flat particles is similar. As a result, this technique has a unique potential for improving the roughness of free-form surfaces, just by arrangement electrical coils, instead of using a robot or special fixture to finish the curved plates.

\subsection{Limitations of TMAF}

Despite the benefits of this method, during the tests, some of the limitations observed. This method can only be used for non-ferrous plates. Iron plates will be magnetized entirely by electromagnetic coils therefore, magnetic abrasive particles will not move on the iron. Coil size is also another limiting factor. By reducing the size of coils, the magnetic force also decreases. Since the magnetic field strength decreases rapidly as the distance increases, this method can only be applied to thin plates and cannot be used for bulk objects because the gap between the coil and the abrasive particles must be small enough that magnetic force can be applied to it and direct them. But in bulk objects, by increasing the distance (between coils and the magnetic particles on the surface) the magnetic force diminishes exponentially and it would be minor for bonding and moving abrasive particles. Hence, this method can only be used for plates.

\section{Conclusion}


In this research, a novel technique in the MAF is demonstrated to improve its potential by using traveling electromagnetic fields. The magnetic field is transmitted by using electrical and electronic techniques and could completely eliminate the need for mechanical machines. The proposed TMAF technique makes it possible to create special and different finishing patterns on the surface of thin sheets simultaneously by controlling the transmitting of the magnetic fields and arranging the magnetic coils. Moreover, the experimental results show that the coil current revealed a significant effect on the surface roughness. And TMAF has the potential to finish free-form surfaces having no special fixture or machine tools, which extends the MAF potential for the automation process and makes it more flexible.

\section{-Ethical Approval}

Ethical approval was not required.

\section{-Consent to Participate}

All participants provided informed consent to participate in the study.

\section{-Consent to Publish}

The publisher has our consent to publish this manuscript including figures and associated data.

\section{-Authors Contributions}

Abbas Moghanizadeh contributed with study design, data collection, data analysis, interpretation of findings and writing of the manuscript.

\section{-Funding}

No funds were used in this study

\section{-Competing Interests}

The authors declare they have no competing interests.

\section{-Availability of data and material}

There are no restrictions on the availability of data and material. 


\section{References}

1. Jayswal, S., V. Jain, and P. Dixit, Modeling and simulation of magnetic abrasive finishing process. The International Journal of Advanced Manufacturing Technology, 2005. 26(5-6): p. 477-490.

2. Singh, D.K., V. Jain, and V. Raghuram, Experimental investigations into forces acting during a magnetic abrasive finishing process. The International Journal of Advanced Manufacturing Technology, 2006. 30(7-8): p. 652-662.

3. Chang, G.-W., B.-H. Yan, and R.-T. Hsu, Study on cylindrical magnetic abrasive finishing using unbonded magnetic abrasives. International Journal of Machine Tools and Manufacture, 2002. 42(5): p. 575-583.

4. Yan, B.-H., et al., Electrolytic magnetic abrasive finishing. International Journal of Machine Tools and Manufacture, 2003. 43(13): p. 1355-1366.

5. Yamaguchi, H. and A.A. Graziano, Surface finishing of cobalt chromium alloy femoral knee components. CIRP Annals, 2014. 63(1): p. 309-312.

6. Verma, G.C., P. Kala, and P.M. Pandey, Experimental investigations into internal magnetic abrasive finishing of pipes. The International Journal of Advanced Manufacturing Technology, 2017. 88(5-8): p. 1657-1668.

7. Ko, S., Y.M. Baron, and J. Park, Micro deburring for precision parts using magnetic abrasive finishing method. Journal of Materials Processing Technology, 2007. 187: p. 19-25.

8. Singh, D.K., V. Jain, and V. Raghuram, Parametric study of magnetic abrasive finishing process. Journal of materials processing technology, 2004. 149(1-3): p. 22-29.

9. Qian, C., et al., A review on magnetic abrasive finishing. The International Journal of Advanced Manufacturing Technology, 2020: p. 1-16.

10. Wang, Y. and D. Hu, Study on the inner surface finishing of tubing by magnetic abrasive finishing. International Journal of Machine Tools and Manufacture, 2005. 45(1): p. 43-49.

11. Yamaguchi, H., T. Shinmura, and M. Sekine, Uniform internal finishing of SUS304 stainless steel bent tube using a magnetic abrasive finishing process. 2005.

12. Vahdati, M. and S. Rasouli, Study of magnetic abrasive finishing on freeform surface. Transactions of the IMF, 2016. 94(6): p. 294-302.

13. Ji, S.M., et al. Comparative study of magnetic abrasive finishing in free-form surface based on different polishing head. in Materials Science Forum. 2011. Trans Tech Publ.

14. Pashmforoush, F. and A. Rahimi, Nano-finishing of BK7 optical glass using 
magnetic abrasive finishing process. Applied optics, 2015. 54(9): p. 21992207.

15. Altareva, G., et al., Use of magnetic abrasive machining for sharpening of medical instrument blades. Biomedical Engineering, 1992. 26(3): p. 148-152.

16. Joshi, R.D., G.S. Brar, and M. Sharma, Effect on magnetic abrasive machining with different range of electrical parameters. Int. J. Emerg. Technol. Adv. Eng, 2014. 9: p. 663-668.

17. Roemer, P.B., R.P. Mallozzi, and Y. Cheng, Optimization of rf transmit and gradient magnetic field imaging using radio frequency and gradient coils. 2011, Google Patents.

18. Rabah, K., et al., Analysis of the magnetic force effect on paramagnetic species. Journal of Electroanalytical Chemistry, 2004. 571(1): p. 85-91.

19. Al-Dulaimi, T. and M.B. Khamesee, A stationary apparatus of magnetic abrasive finishing using a rotating magnetic field. Microsystem Technologies, 2017. 23(11): p. 5185-5191.

\section{Figure captions}

Figure 1. The schematic of transmitting magnetic field from one coil to another that lead to moving magnetic and magnetic abrasive particles (A to D).

Figure 2. The schematic of electrical coil and its dimensions.

Figure 3. The schematic of magnetic force applied on magnetic abrasive particles (coil No. 1 is turned on and exerts a vertical magnetic force on the abrasive particles, then Coil No. 1 is turned off and coil No. 2 is turned on, causing a horizontal force to be applied to the abrasive magnetic particles. And the result of the forces will lead to the movement of magnetic abrasive particles and eventually surface wear.

Figure 4. Atomic force microscopy (AFM) images, A: initial surface, B: after 1 hour TMAF process, C: after 2 hour TMAF process, D: after 3 hour TMAF process (electrical current is $0.5 \mathrm{~A}$, particle speed is $30 \mathrm{~mm} / \mathrm{s}$ for all cases).

Figure 5. Atomic force microscopy (AFM) images, A: initial surface, B: electrical current is $0.25 \mathrm{~A}$ in coils during TMAF process, $\mathrm{C}$ : electrical current is $0.5 \mathrm{~A}$ in coils during TMAF process, $\mathrm{D}$ : electrical current is $0.75 \mathrm{~A}$ in coils during TMAF process (time of process is 2 hours, particle speed is $30 \mathrm{~mm} / \mathrm{s}$ for all cases). 
Figure 6. The arrangement of electrical coils for creating finishing in the form of $\mathrm{L}$ pattern.

Figure 7. The effect of TMAF on the surface roughness in two perpendicular directions (path A-B and path B-C) (finishing time is 1 hour, with particle speed of $20 \mathrm{~mm} / \mathrm{s}$ ) (error bars represent standard deviation of five independent experiments).

Figure 8. The effect of TMAF on the surface roughness in two perpendicular directions (path A-B and path B-C) (finishing time is 2 hours, with particle speed of $30 \mathrm{~mm} / \mathrm{s}$ ) (error bars represent standard deviation of five independent experiments).

Figure 9. The effect of TMAF on the surface roughness in two perpendicular directions (path A-B and path B-C) (finishing time is 3 hours, with particle speed of $40 \mathrm{~mm} / \mathrm{s}$ ) (error bars represent standard deviation of five independent experiments).

Figure 10. The effect of TMAF on the surface roughness in two perpendicular directions with different magnetic fields value (electrical current of coils in path A$\mathrm{B}$ is $0.25 \mathrm{~A}$, and electrical current of coils in path $\mathrm{B}-\mathrm{C}$ is $0.5 \mathrm{~A}$ ). (finishing time is 1 hours) (error bars represent standard deviation of five independent experiments).

Figure 11. The effect of TMAF on the surface roughness in two perpendicular directions with different magnetic fields value (electrical current of coils in path A$\mathrm{B}$ is $0.25 \mathrm{~A}$, and electrical current of coils in path $\mathrm{B}-\mathrm{C}$ is $0.75 \mathrm{~A}$ ). (finishing time is 1 hours) (error bars represent standard deviation of five independent experiments).

Figure 12. The schematic of TMAF in free-form plate

\section{Tables}

Table 1: The effect of TMAF parameters on the surface roughness in flat and curve surface 
Figures

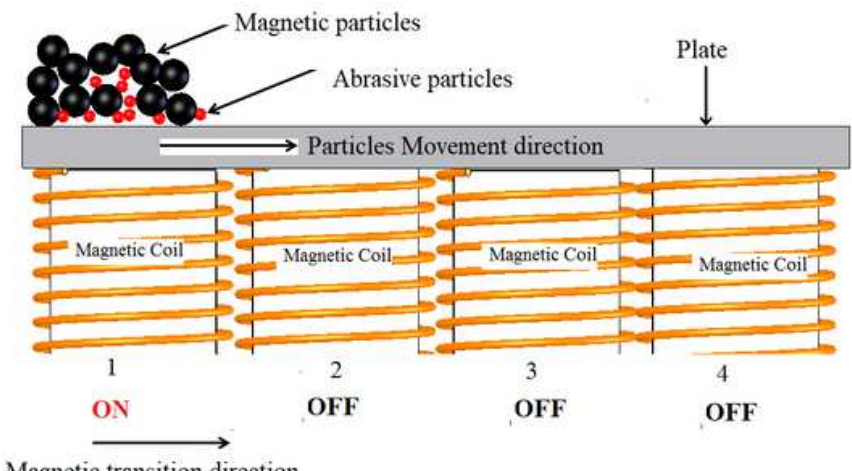

Magnetic transition direction

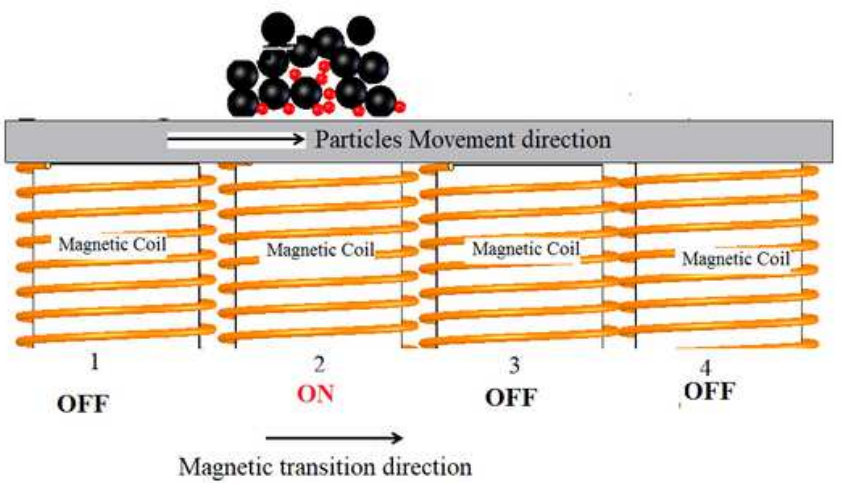

A

B

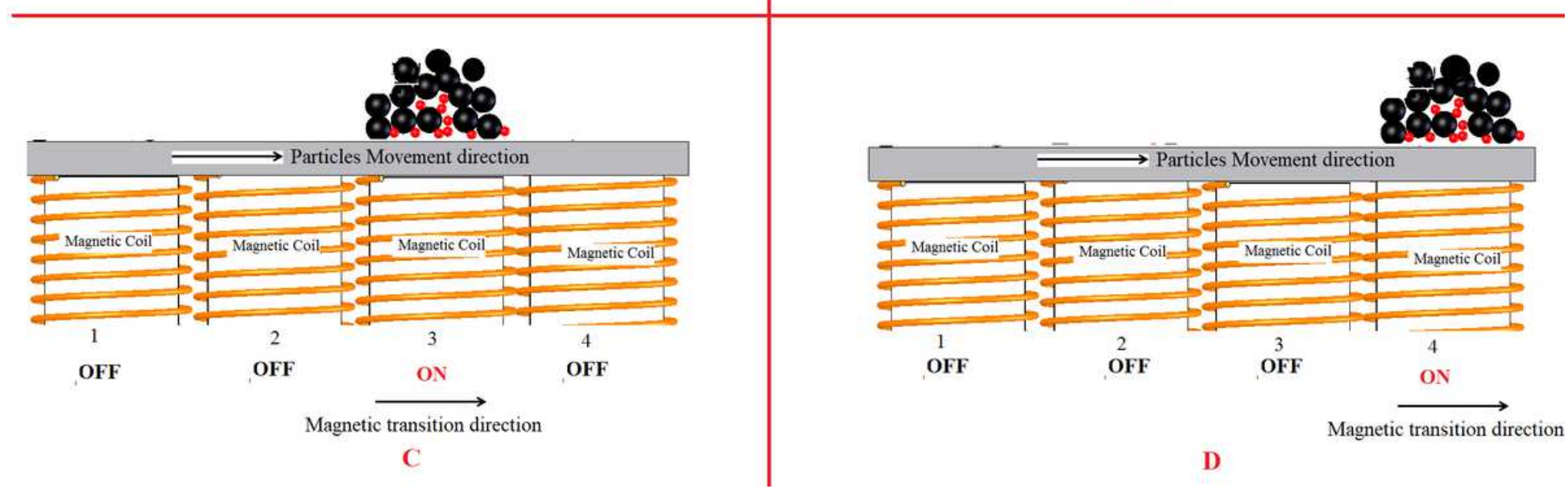

Figure 1

The schematic of transmitting magnetic field from one coil to another that lead to moving magnetic and magnetic abrasive particles (A to D). 

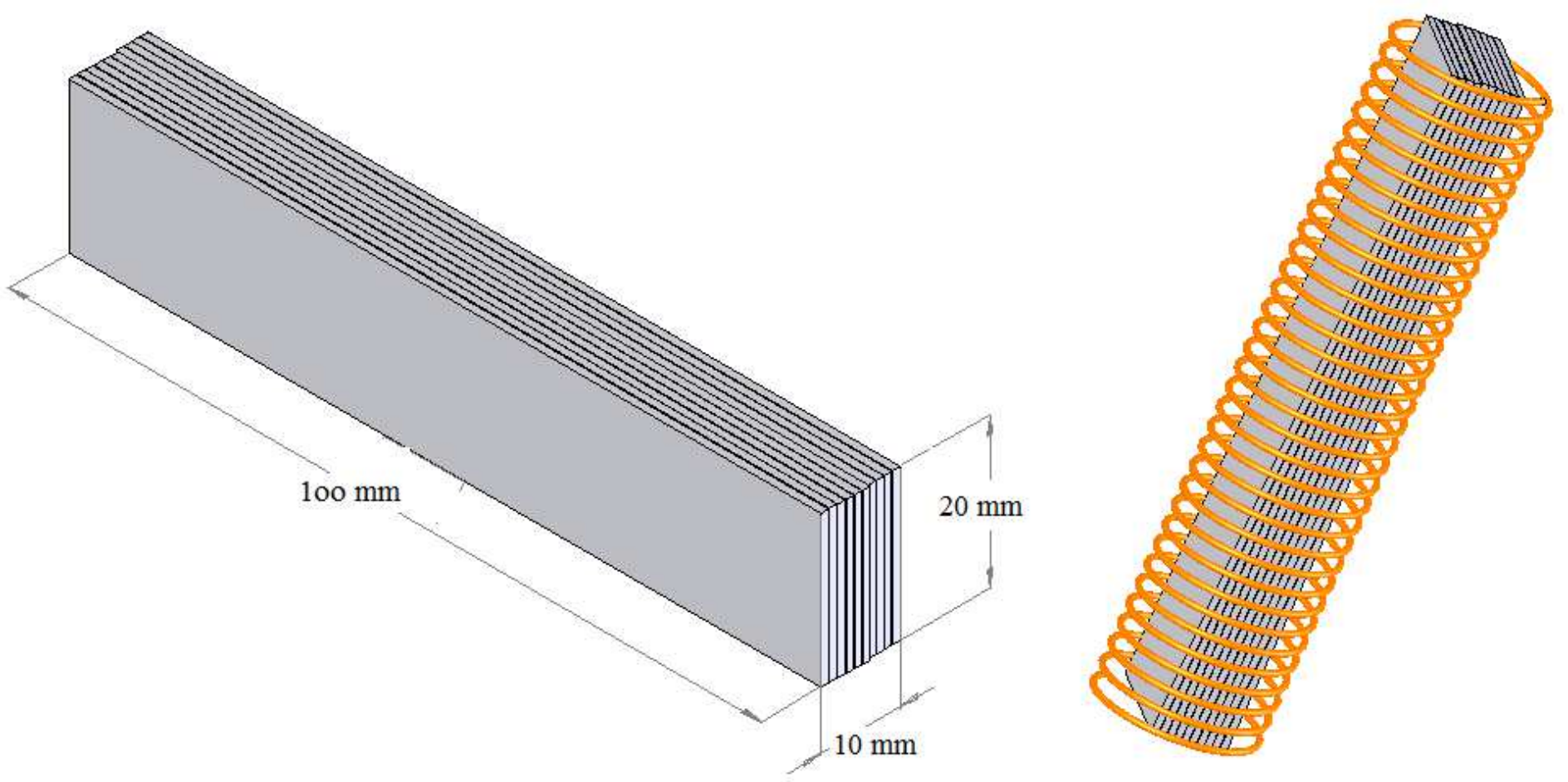

Figure 2

The schematic of electrical coil and its dimensions. 

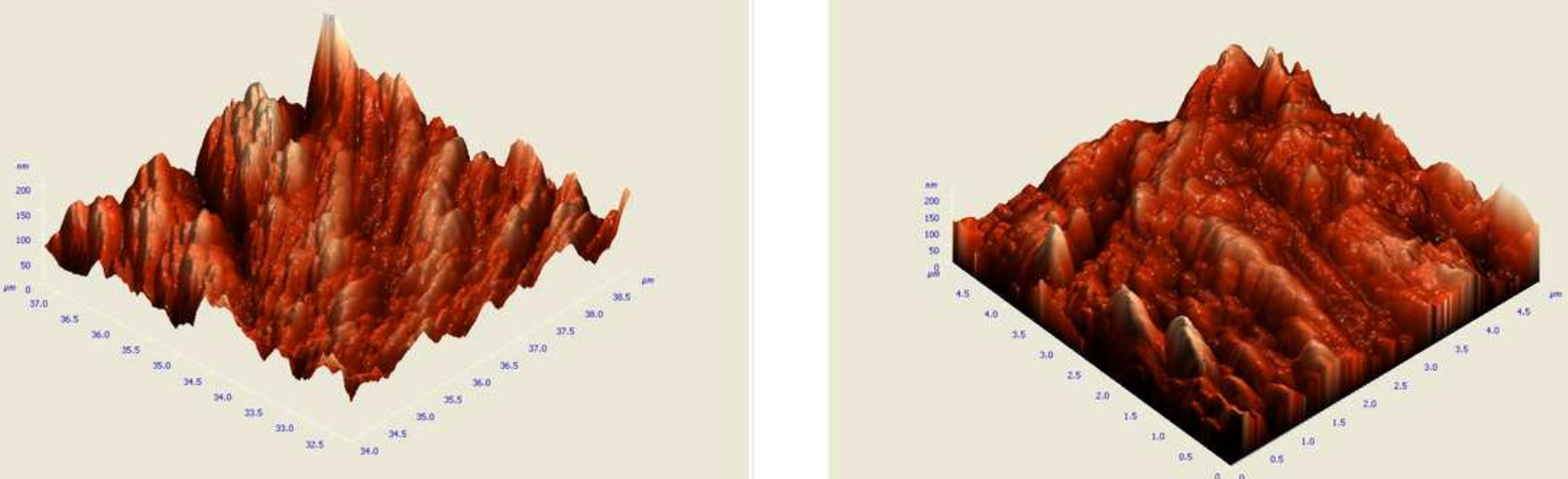

A

B

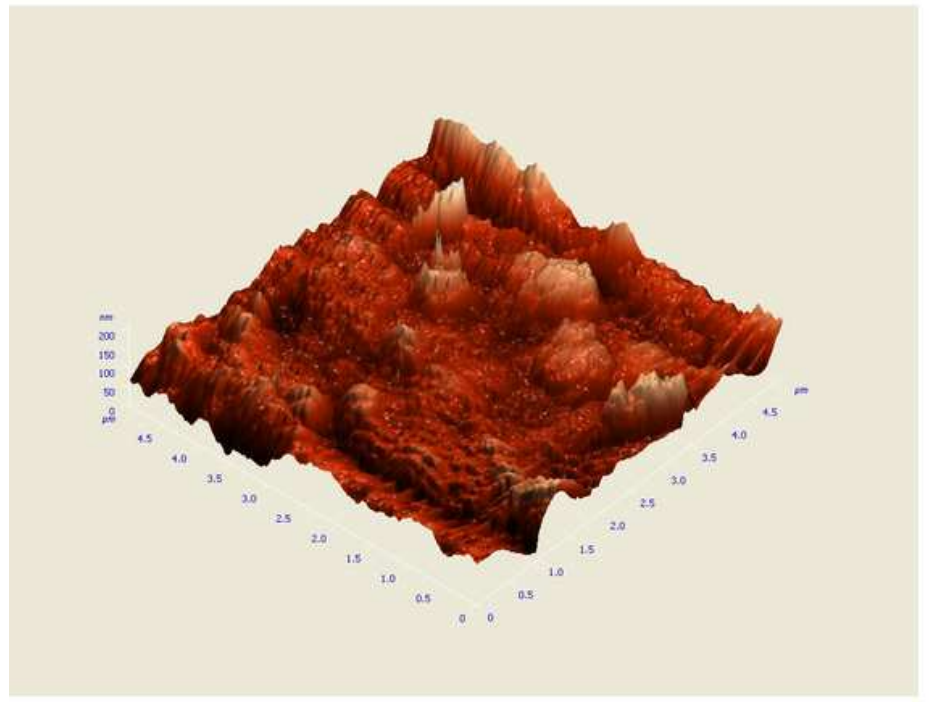

C

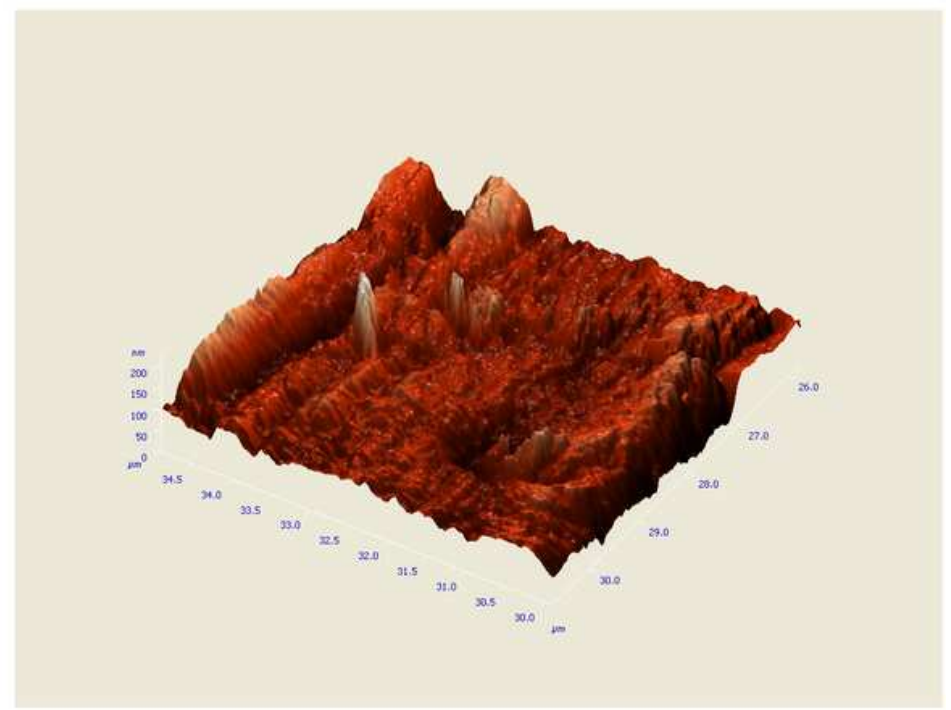

D

Figure 3

The schematic of magnetic force applied on magnetic abrasive particles (coil No. 1 is turned on and exerts a vertical magnetic force on the abrasive particles, then Coil No. 1 is turned off and coil No. 2 is turned on, causing a horizontal force to be applied to the abrasive magnetic particles. And the result of the forces will lead to the movement of magnetic abrasive particles and eventually surface wear. 


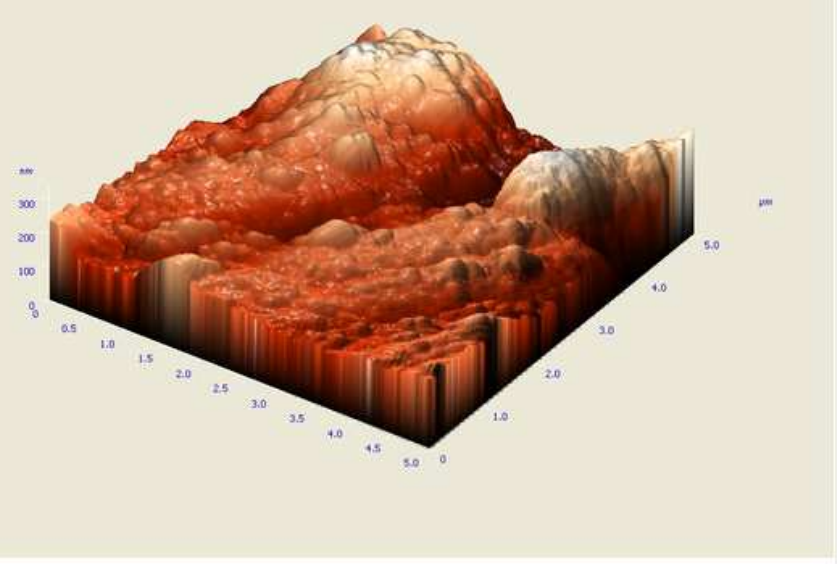

A

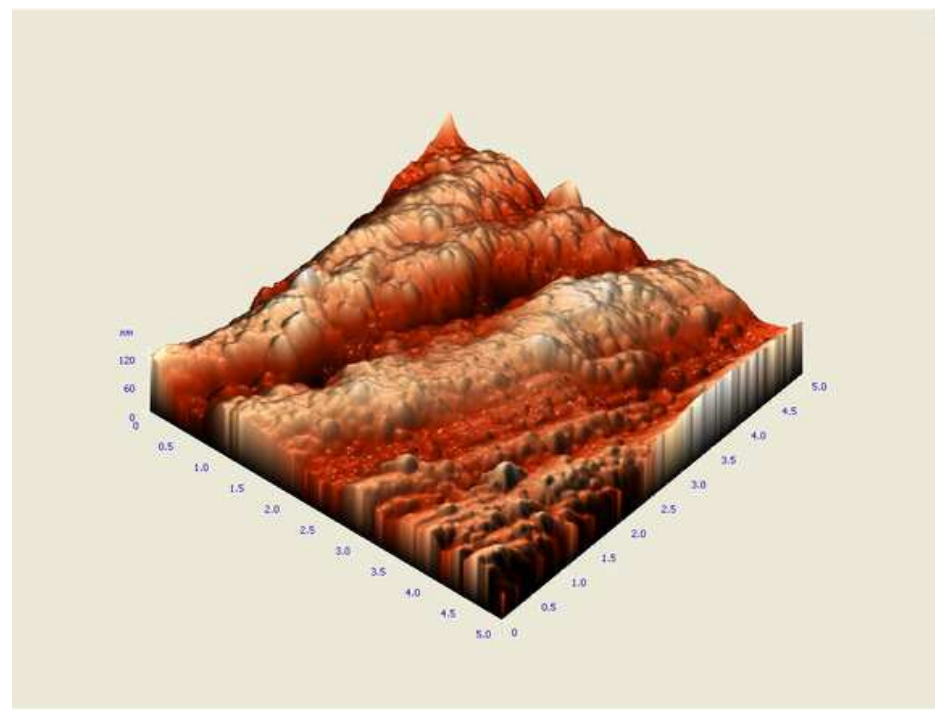

C

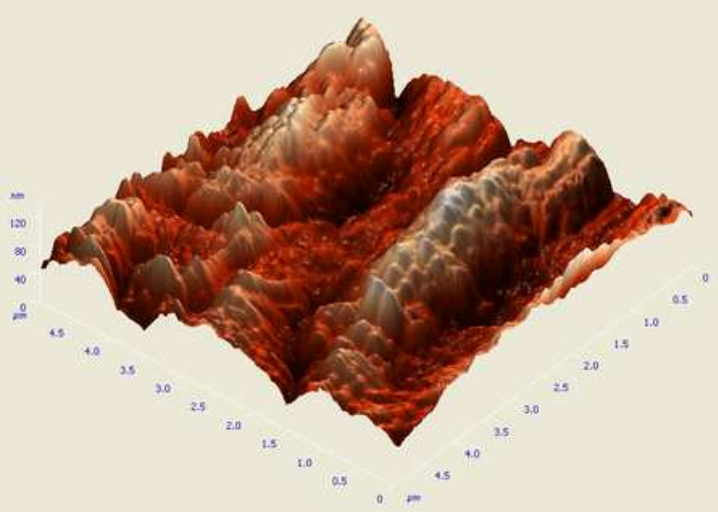

B

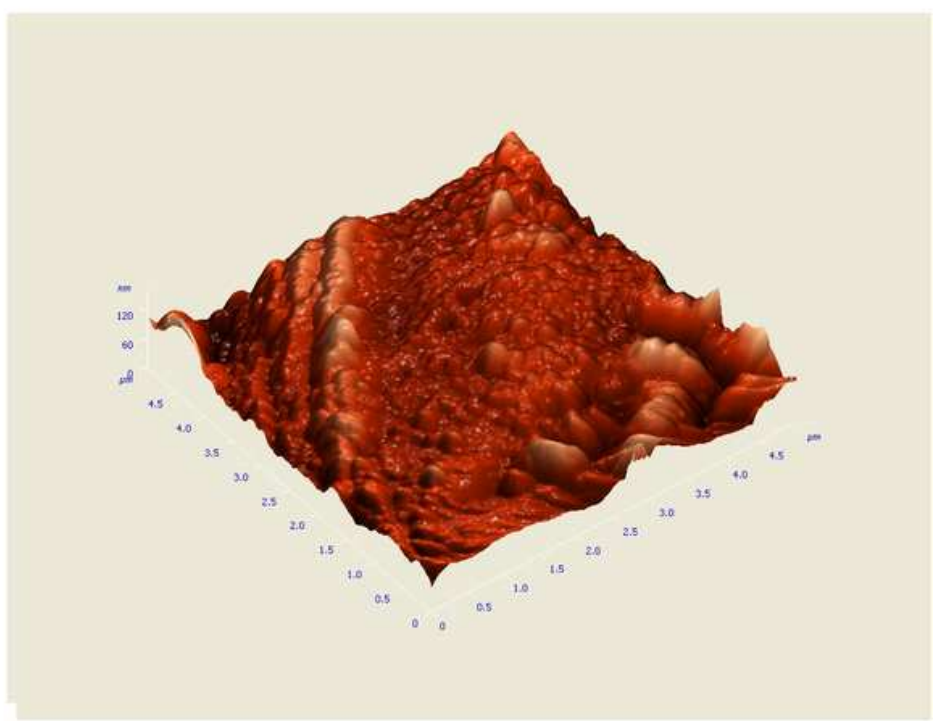

D

\section{Figure 4}

Atomic force microscopy (AFM) images, A: initial surface, B: after 1 hour TMAF process, C: after 2 hour TMAF process, D: after 3 hour TMAF process (electrical current is $0.5 \mathrm{~A}$, particle speed is $30 \mathrm{~mm} / \mathrm{s}$ for all cases). 


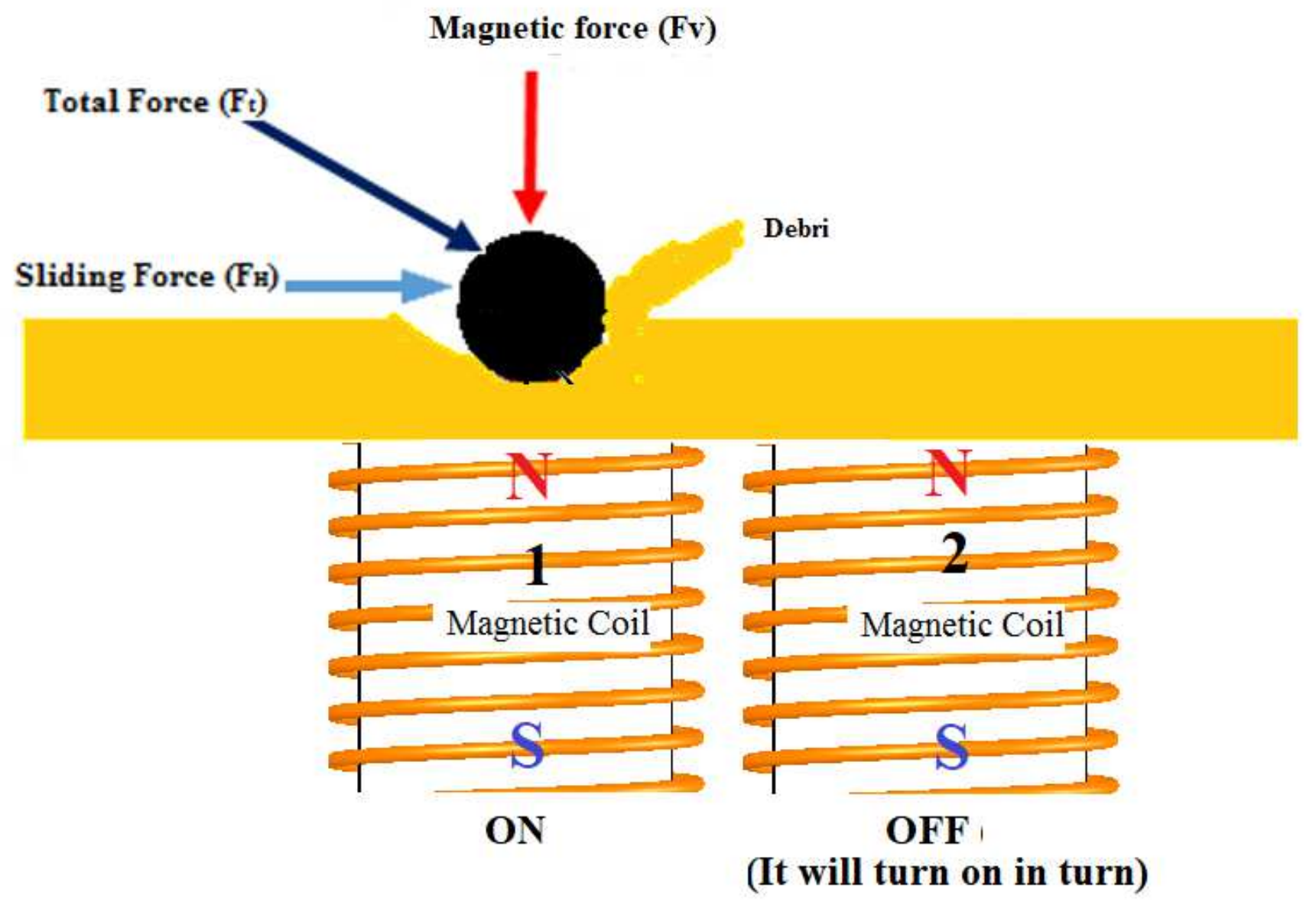

Figure 5

Atomic force microscopy (AFM) images, A: initial surface, B: electrical current is $0.25 \mathrm{~A}$ in coils during TMAF process, $C$ : electrical current is $0.5 \mathrm{~A}$ in coils during TMAF process, $\mathrm{D}$ : electrical current is $0.75 \mathrm{~A}$ in coils during TMAF process (time of process is 2 hours, particle speed is $30 \mathrm{~mm} / \mathrm{s}$ for all cases). 


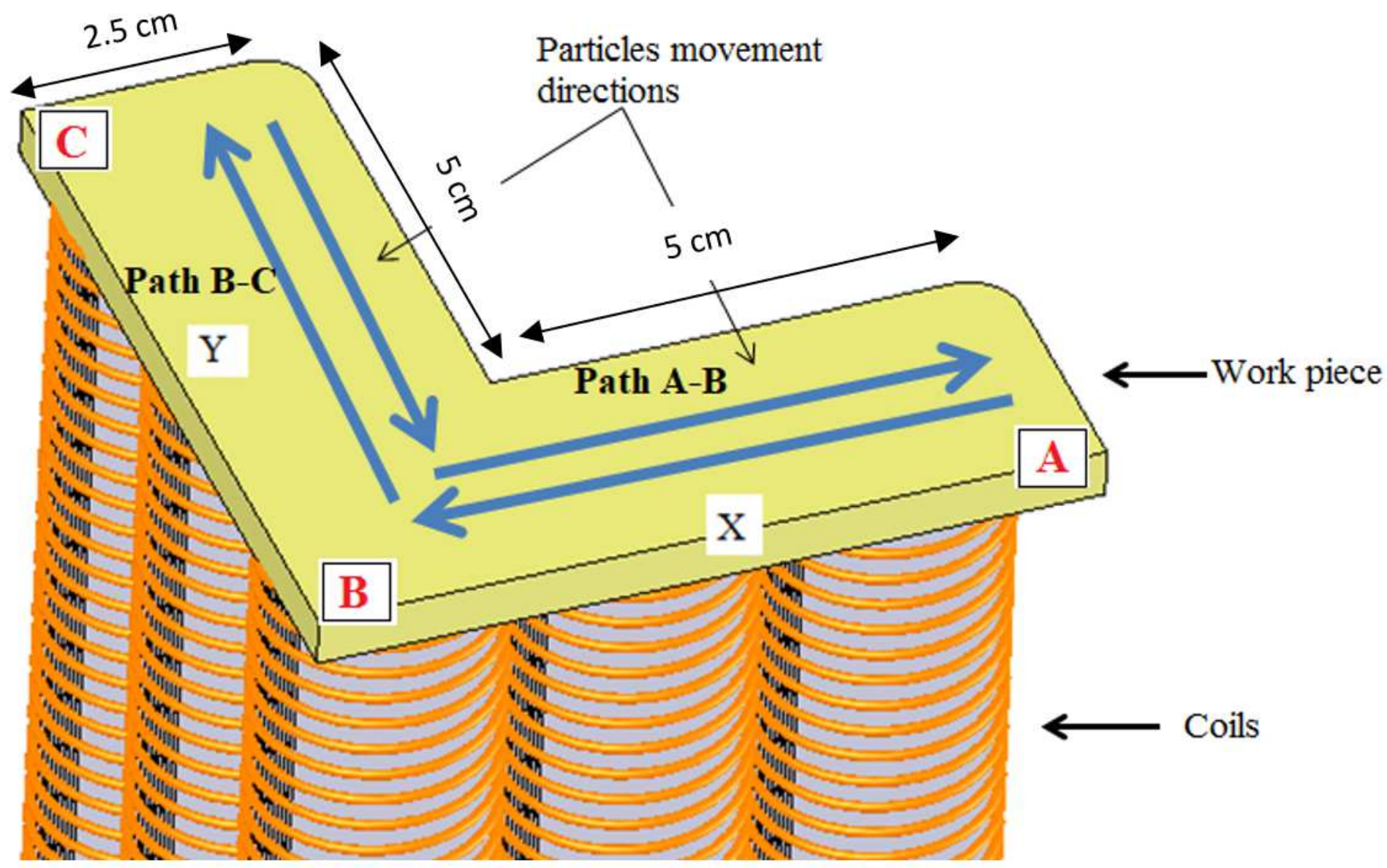

Figure 6

The arrangement of electrical coils for creating finishing in the form of $L$ pattern. 


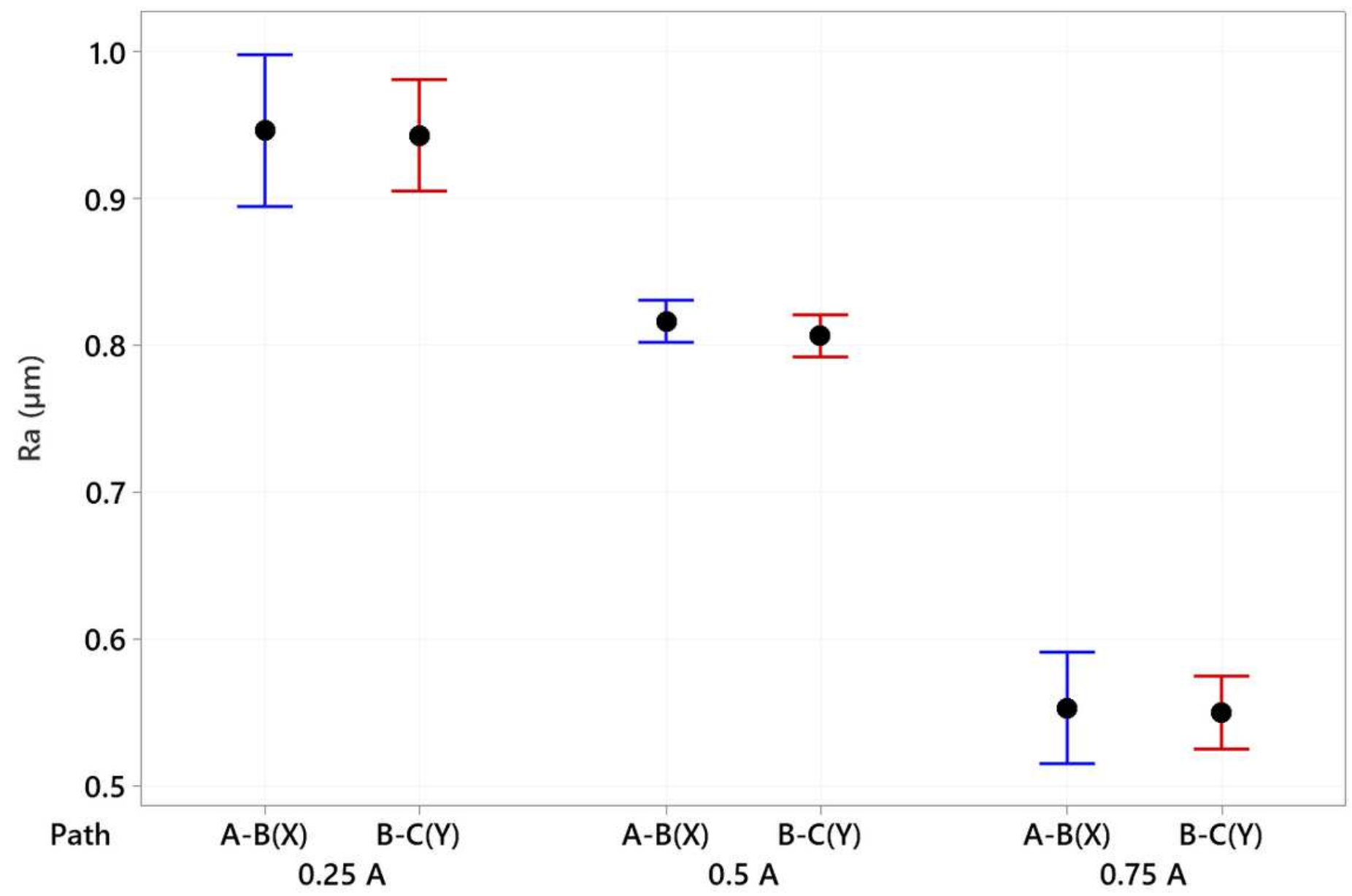

Figure 7

The effect of TMAF on the surface roughness in two perpendicular directions (path A-B and path B-C) (finishing time is 1 hour, with particle speed of $20 \mathrm{~mm} / \mathrm{s}$ ) (error bars represent standard deviation of five independent experiments). 


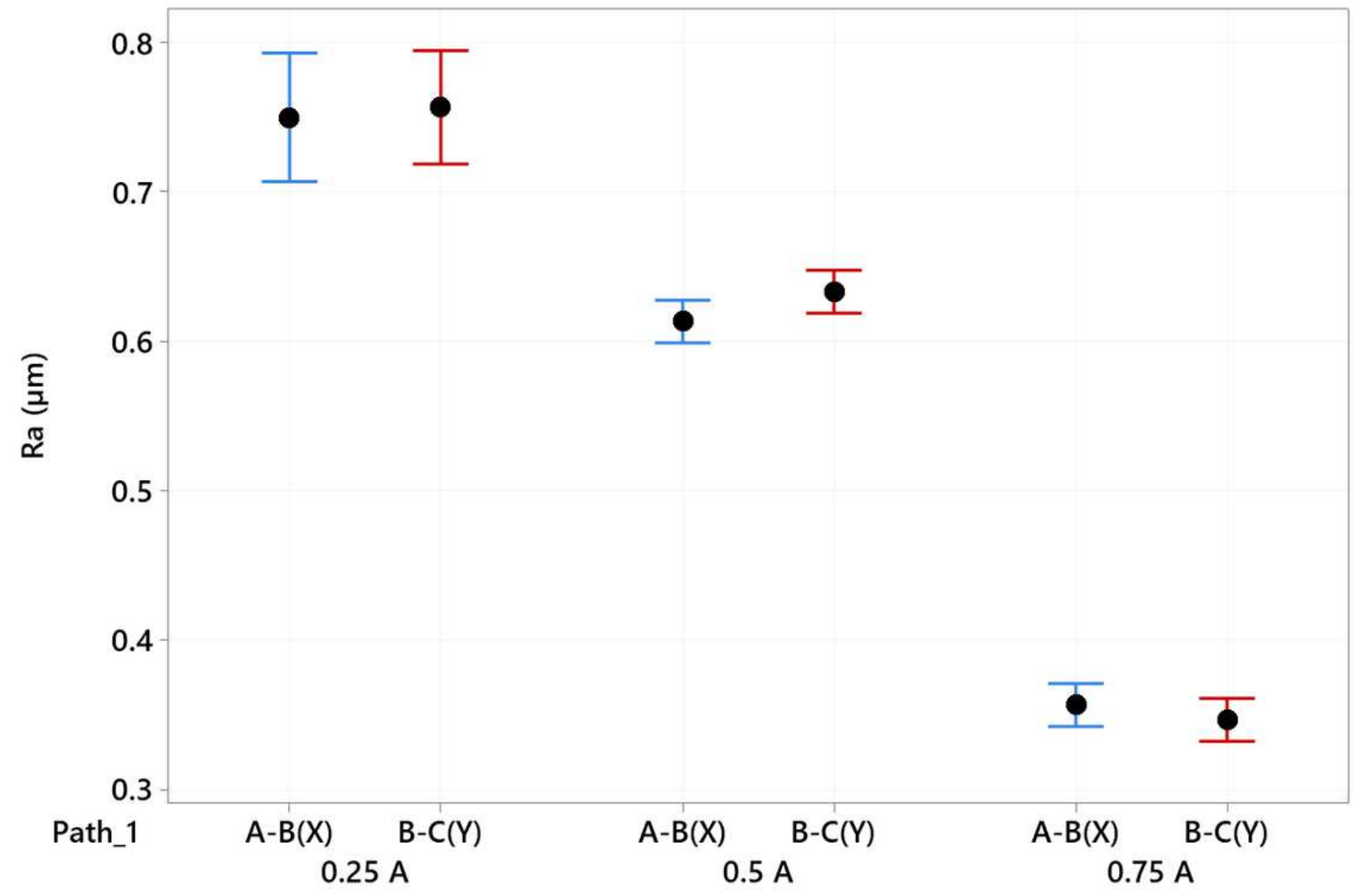

Figure 8

The effect of TMAF on the surface roughness in two perpendicular directions (path A-B and path B-C) (finishing time is 2 hours, with particle speed of $30 \mathrm{~mm} / \mathrm{s}$ ) (error bars represent standard deviation of five independent experiments). 


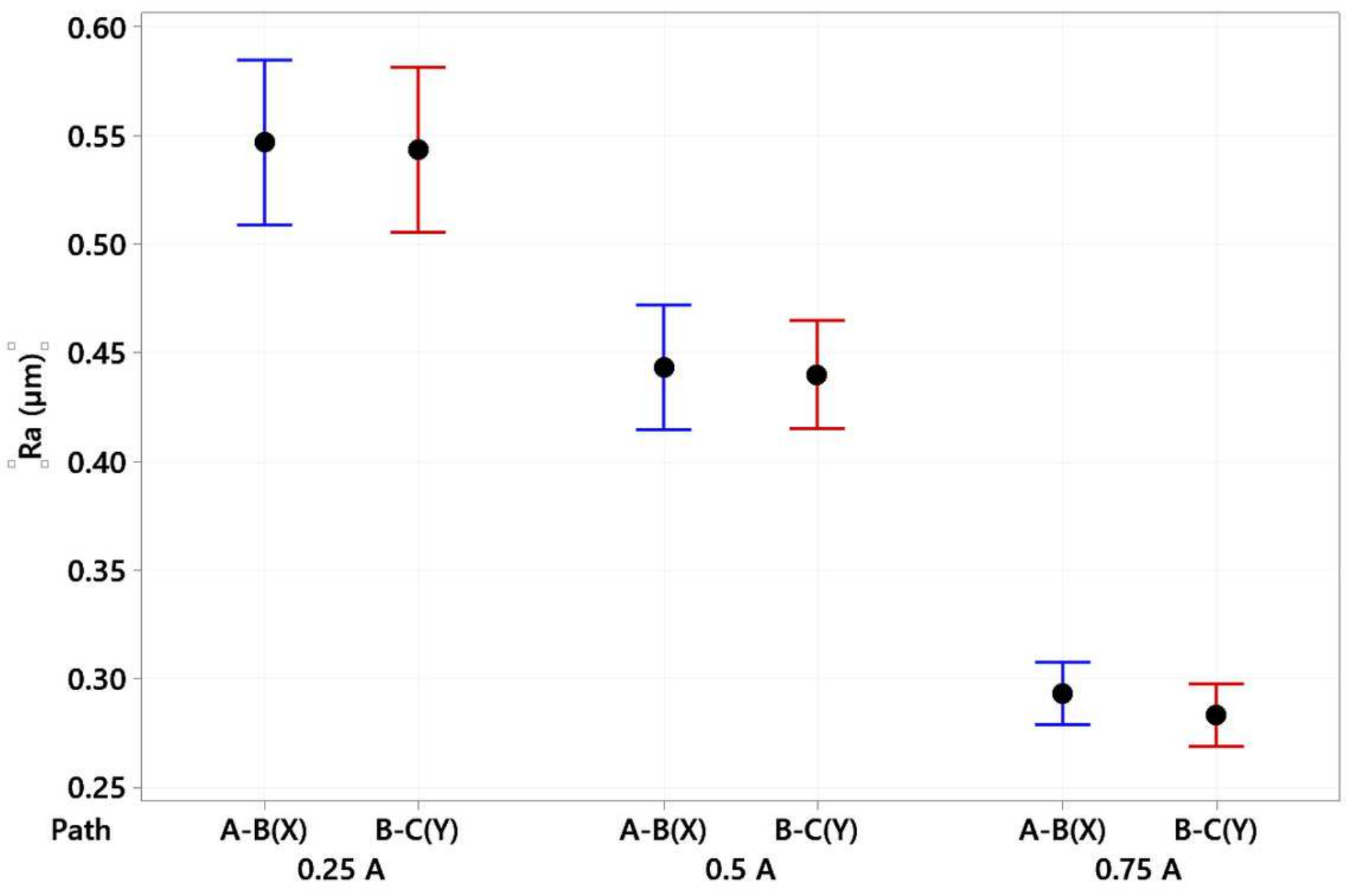

Figure 9

The effect of TMAF on the surface roughness in two perpendicular directions (path A-B and path B-C) (finishing time is 3 hours, with particle speed of $40 \mathrm{~mm} / \mathrm{s}$ ) (error bars represent standard deviation of five independent experiments). 


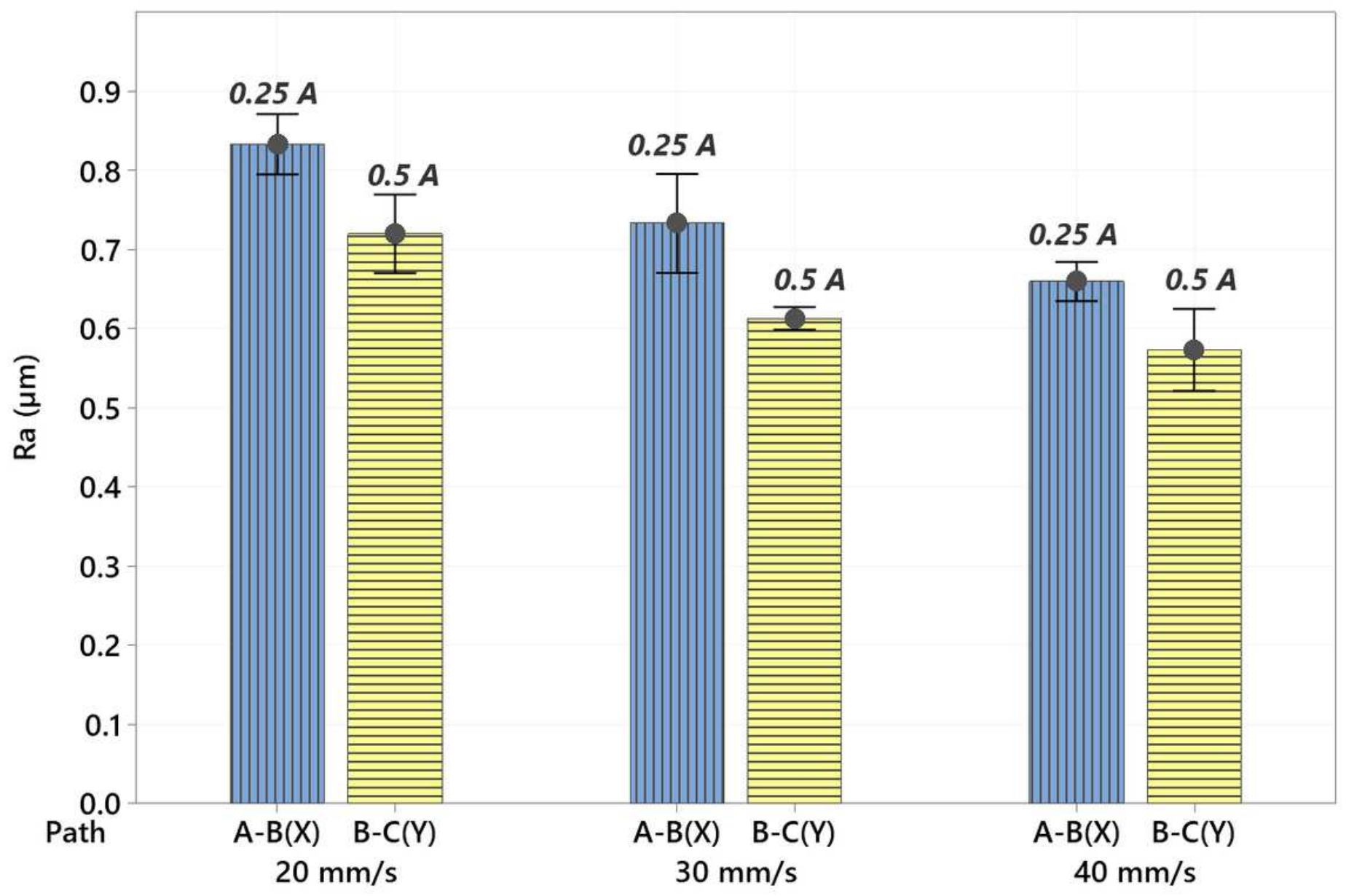

Figure 10

The effect of TMAF on the surface roughness in two perpendicular directions with different magnetic fields value (electrical current of coils in path A-B is $0.25 \mathrm{~A}$, and electrical current of coils in path B-C is $0.5 \mathrm{~A})$. (finishing time is 1 hours) (error bars represent standard deviation of five independent experiments). 


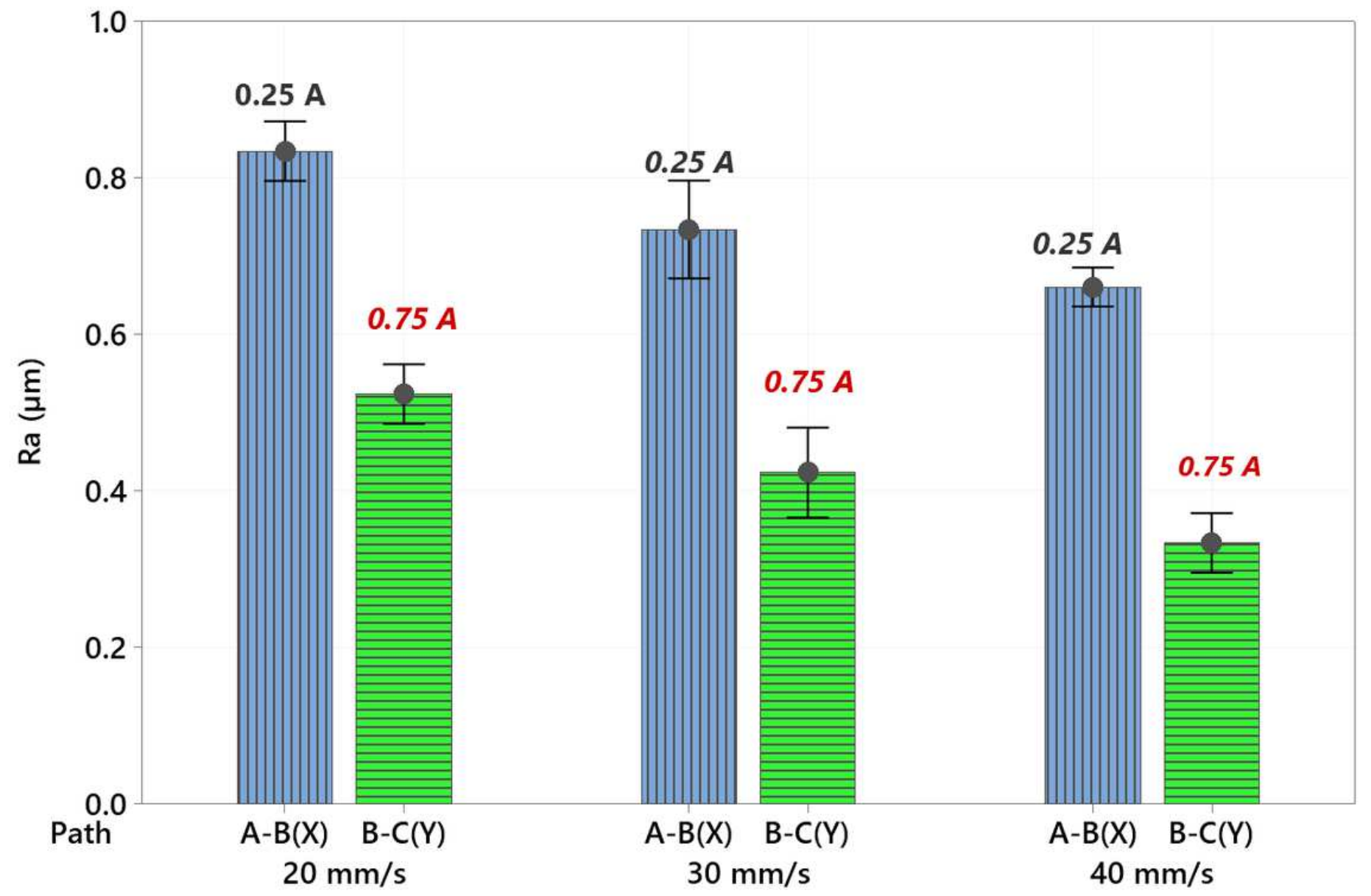

Figure 11

The effect of TMAF on the surface roughness in two perpendicular directions with different magnetic fields value (electrical current of coils in path A-B is $0.25 \mathrm{~A}$, and electrical current of coils in path $\mathrm{B}-\mathrm{C}$ is $0.75 \mathrm{~A}$ ). (finishing time is 1 hours) (error bars represent standard deviation of five independent experiments).

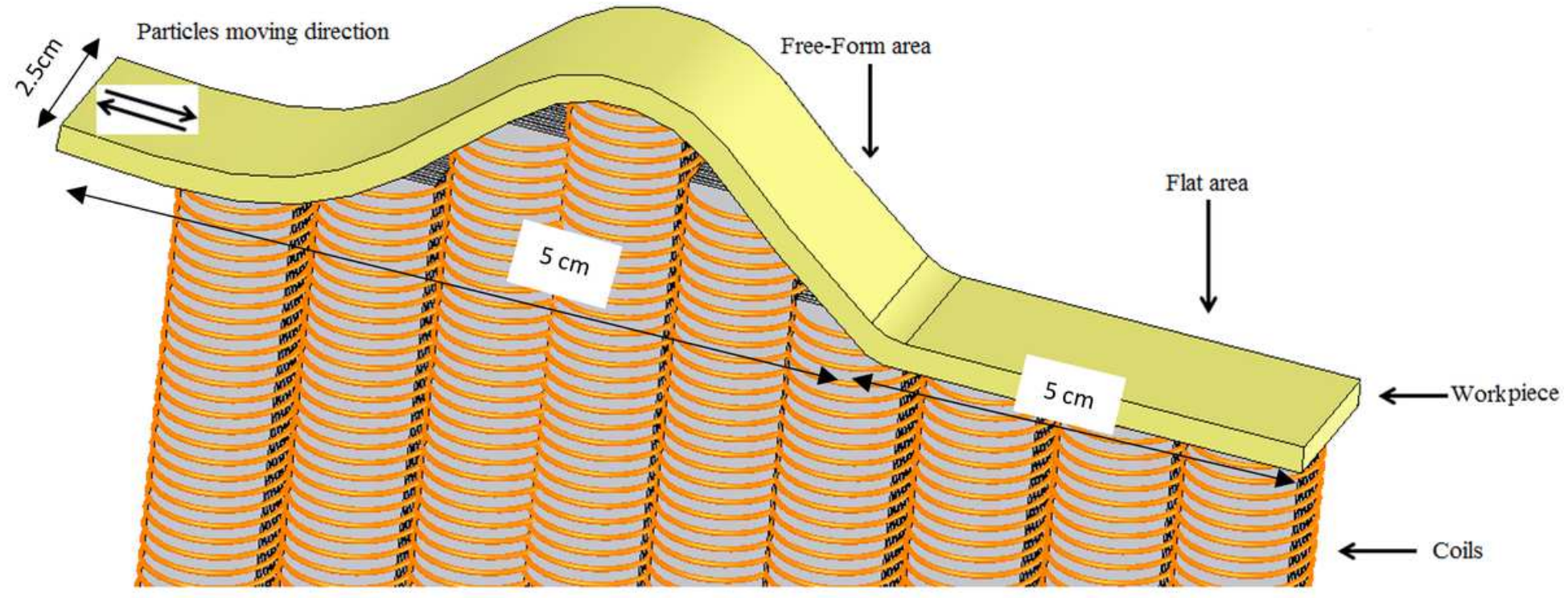


Figure 12

The schematic of TMAF in free-form plate 